\title{
À la recherche d'une citoyenneté globale. L'expérience des adolescents migrants en Europe
}

Searching for a Global Citizenship. Experience of Migrant Minors in Europe

En busca de una ciudadanía global. La experiencia de los menores migrantes en

Europa

Francesco Vacchiano

\section{CpenEdition}

Journals

Édition électronique

URL : https://journals.openedition.org/remi/6761

DOI : $10.4000 /$ remi. 6761

ISSN : $1777-5418$

Éditeur

Université de Poitiers

Édition imprimée

Date de publication : 1 mars 2014

Pagination : 59-81

ISBN : 979-10-90426-21-4

ISSN : 0765-0752

Référence électronique

Francesco Vacchiano, «À la recherche d'une citoyenneté globale. L'expérience des adolescents migrants en Europe », Revue européenne des migrations internationales [En ligne], vol. $30-\mathrm{n}^{\circ} 1$ | 2014, mis en ligne le 01 mars 2017, consulté le 14 avril 2022. URL : http://journals.openedition.org/remi/ 6761 ; DOI : https://doi.org/10.4000/remi.6761 


\section{À la recherche d'une citoyenneté globale. L'expérience des adolescents migrants en Europe}

\section{Francesco Vacchiano ${ }^{1}$}

\section{Introduction}

Ces vingt dernières années, les enfants et les adolescents migrants ont cessé d'être des figures secondaires, subordonnées à celles de parents, pour assumer un rôle de premier plan alors qu'ils expérimentaient des nouvelles formes de mobilité relativement indépendantes. Ce phénomène, qui a été observé dans plusieurs pays tant sur le plan quantitatif que qualitatif, a conduit en Europe à la création d'une nouvelle catégorie juridique, celle de " mineur non accompagné ", attribuée à " tous les nationaux de pays tiers de moins de 18 ans qui entrent dans le territoire des États membres sans être accompagnés d'un adulte qui soit responsable d'eux par effet de la loi ou de fait, et tant qu'ils ne soient pas effectivement à charge d'une telle personne ${ }^{2}$.

J'ai rencontré le premier " mineur non accompagné " en 1998 au Centre Frantz Fanon de Turin ${ }^{3}$ où je travaillais en tant qu'ethnopsychologue. Cette jeune fille âgée de seize ans, originaire de Roumanie, était arrivée en Italie par l'intermédiaire d'un réseau de traite qui acheminait ses victimes vers la prostitution forcée. Alors que mon intervention consistait à I'aider dans sa difficile élaboration des violences subies, je notais que les gens qui l'avaient " appâtée ", dans une discothèque de Bucarest, avaient principalement profité de son désir de partir, sous-tendu par l'image d'un " ailleurs " fascinant au fort pouvoir évocateur.

1 PhD, ICS-UL (Instituto de Ciências Sociais - Universidade de Lisboa), Av. Professor Aníbal de Bettencourt 9, 1600-189 Lisboa, Portugal ; francesco.vacchiano@ics.ul.pt

2 Résolution du Conseil de I'Union européenne du 26 juin 1997 concernant les mineurs non accompagnés ressortissants de pays tiers, art. 1.

3 Le Centre Frantz Fanon, ouvert à Turin en 1996, est un centre clinique, de recherche appliquée et de formation, dédié à la santé mentale des migrants. Il est animé par une équipe multidisciplinaire composée de psychologues, de psychiatres, d'anthropologues et de médiateurs culturels (Beneduce, Frigessi, Taliani, et Vacchiano, 2000 ; Beneduce, 2004 ; Taliani et Vacchiano, 2006). 
Depuis cette première expérience, j'ai poursuivi ma recherche 4 à l'écoute de nombreux migrants - jeunes ou pas - qui décrivaient leur désir d'Europe comme un passage obligé pour leur accomplissement personnel, un achèvement perçu comme inaccessible dans leur pays d'origine. Qu'il s'agisse du Maroc, du Nigéria, de l'Afghanistan, du Sénégal ou d'autres pays encore, et quelle que soit la spécificité de leur profil administratif (" victimes de traite ", " demandeurs d'asile ", "réfugiés ", " mineurs non accompagnés ", "isolés ", " séparés ", etc. $)^{5}$, la majorité des personnes que je rencontrais exprimait un profond désir de changement, qui donnait lieu à des parcours migratoires souvent très risqués.

De quel désir s'agit-il ? Dans l'introduction à un ouvrage collectif sur l'enfance et l'adolescence en Afrique, De Boeck et Honwana définissent les jeunes comme " a window to understand the wider socio-political and economical transformations in Africa and explore how these processes of change shape and are shaped by youth " (De Boeck et Honwana, $2005: 1)^{6}$. Selon ces auteurs, les aspirations et les attitudes des jeunes sont le reflet de processus sociaux et politiques au niveau global et local. Comme ils l'observent, " children and youth are the main actors in the new informal economies and in the processes of globalisation, as well as in the definition of local, alternative forms of mobility [...] at the frontline in movements which incorporate the expectations and promises of millennial capitalism " (De Boeck et Honwana, 2005 : 1)7. Dans cette logique, nous pouvons nous demander quelles sont les transformations impliquées par " la mise en mouvement " de ces adolescents et quel sens donner à leurs aspirations. Que nous dit donc ce phénomène sur la situation des enfants et des adolescents dans les pays d'origine et dans le monde contemporain en général ? En admettant que ces nouvelles formes de mobilité puissent être considérées comme historiquement spécifiques - c'est là l'hypothèse que je discuterai -, il convient de les présenter brièvement.

Bien que le nouveau rôle des adolescents dans la migration internationale soit une question largement débattue, peu d'auteurs en ont tenté une lecture

4 Dans mon doctorat en anthropologie, réalisé à l'université de Turin de 2002 à 2008, j'ai travaillé sur la relation entre désir et norme dans l'expérience de la migration " irrégulière " des jeunes migrants entre le Maroc et l'Italie. Depuis 2010, je poursuis des recherches sur les migrations, la jeunesse postcoloniale et la subjectivité dans la région méditerranéenne dans le cadre d'un projet postdoctoral financé par la Fundação para a Ciência e a Tecnologia du Portugal, actuellement en cours à I'Instituto de Ciências Sociais de l'université de Lisbonne.

5 Le rapport entre les causes de la mobilité contemporaine et les profils cités n'est pas direct. Ces profils sont définis administrativement en identifiant - et sélectionnant - des conditions d'admissibilité selon un critère de régularité ou irrégularité. Pour une discussion, voir Bakewell (2008b) ; Fassin (2011) ; Long (2013).

6 « Une fenêtre pour comprendre les plus amples transformations en Afrique et explorer comment ces processus de changement forment et sont formés par les jeunes " [ma traduction].

7 « Les jeunes sont les acteurs des nouvelles économies informelles et des processus de globalisation, ainsi que dans la définition des formes locales et alternatives de modernité " [ma traduction]. 
par rapport aux changements contemporains du lien social et de la subjectivité ${ }^{8}$. La plupart des contributions a été consacrée à la description du phénomène dans des contextes spécifiques de réception, en particulier vis-à-vis des importants problèmes de prise en charge institutionnelle et de protection de l'enfance (Etiemble, 2008 ; Frechaut, 2007 ; Giordano, 2009 ; Giovannetti, 2009 ; Quiroga, Alonso et Sòria, 2010 ; Suárez-Navaz et Jiménez Alvarez, 2011) ${ }^{9}$. Certaines de ces études explorent les dimensions de risque liées à la condition de " mineur isolé " - déviance, toxicomanie, prostitution - et ses implications pour la prise en charge (Derluyn et Broekaert, 2007 ; Dipartimento Giustizia Minorile, 2008 ; Sourander, 1998).

La mise en œuvre progressive de politiques migratoires restrictives et leur impact sur la qualité de la protection de l'enfance immigrée ont généré une grande production consacrée à l'analyse et à l'évaluation des politiques de réception. Ces travaux, dont certains ont été réalisés avec des méthodologies comparatives rigoureuses, ont mis en évidence les limites des systèmes de réception (Carchedi, 2009 ; Fangen, Fossan et Mohn, 2010 ; Giovannetti, 2008 ; Laiz Moreira, 2013 ; Senovilla Hernández, 2007 et 2012 ; Sigona, 2011). Suite aux fréquents cas de non respect des droits des mineurs migrants par les administrations publiques en Europe - c'est le cas par exemple des renvois à la frontière, des retours forcés, des preuves répétées et arbitraires pour la détermination de l'âge, de la détention, etc. - de nombreuses contributions ont porté sur la dénonciation des violations des droits et sur leurs conséquences ${ }^{10}$. Ces recherches mettent en évidence la contradiction entre les discours sur les droits de l'enfance et les pratiques de contrôle des flux migratoires, qui souvent aboutissent à une polarisation du débat public autour des termes opposés et complémentaires, de la victimisation et de la criminalisation (Furia, 2012 ; Parousel, 2011).

Malgré cette riche production, peu d'auteurs ont exploré non seulement les conditions matérielles, mais aussi les formes de représentation et de projection subjective, au niveau individuel ou familial, à travers lesquelles le projet migratoire des enfants prend forme ${ }^{11}$. Ces contributions soulignent l'importance du processus de comparaison entre la situation personnelle et familiale et les caractéristiques d'un "bien-être " imaginé comme mode de vie souhaitable. $C^{\prime}$ est sur cette articulation, entre conditions réelles et projections imaginaires du monde et de l'avenir, que mon analyse se centrera, en essayant de comprendre la relation entre construction du sujet " moderne " et mobilité dans le monde

8 Parmi les contributions les plus intéressantes je souligne les travaux de Paula Fass (2003 et 2005) sur l'émergence du paradigme de l'enfance, la mondialisation et la mobilité des enfants, de Peter Stearns (2005) sur le changement social et son impact sur I'enfance et de Liliana Suárez Navaz (2006) sur la transformation des relations entre sexes et générations et sur la " crise de la dépendance " induites par les mutations capitalistes et néo-capitalistes. Whitehead et Hashim proposent plutôt une lecture et une première explication de la migration indépendante des enfants comme une réponse aux crises structurelles dans les contextes d'origine, liés à des phénomènes de conflit, de crise économique et d'épidémies (Whitehead et Hashim, 2005). Ma contribution discute cette perspective avec pour objectif de la complexifier.

9 Je me limite à signaler certains travaux qui illustrent bien les thèmes des débats dans les différents pays concernés.

10 Parmi les nombreux matériaux disponibles, je signale Human Rights Watch (1997, 2002, 2008, 2009, 2012 et 2013) ; SOS Racismo y Colectivo Al Jaima (2005).

11 Voir notamment Unicef Maroc (2005) ; Vacchiano (2008) ; Giordano (2011) ; Volpicelli (2012). 
contemporain. Les adolescents et les jeunes sont en ce sens, comme l'indiquent De Boeck et Honwana, les protagonistes d'un processus de signification du monde, de négociation de l'appartenance et de revendication de citoyenneté qui trouve dans l'accès à la mobilité l'une de ses dimensions les plus considérables.

Dans cet article, je propose donc d'analyser la mobilité des mineurs en partant de l'examen des motivations et des discours sur soi et sur le monde exprimés par des adolescents de différentes nationalités que j'ai rencontrés au cours des quinze dernières années entre I'Italie, le Maroc, I'Espagne, la France et la Tunisie. J'ai eu la possibilité de recueillir leurs paroles dans une pluralité de situations en contexte : dans des centres d'accueil en tant que psychologue, mais aussi dans les quartiers d'origine et les zones de frontière au cours de mes recherches de terrain.

Les témoignages et les récits de vie que je cite ne représentent qu'une petite partie des situations sur lesquelles j'ai travaillé et qui ont inspiré la réflexion que je propose ici. J'essaie de démontrer qu'au-delà des conditions matérielles spécifiques des contextes d'origine - qui me paraissent toutefois importants et que j'ai analysé dans d'autres travaux (Vacchiano et Jiménez, 2012 ; Vacchiano, 2008, 2010 et 2012) - une toile de fond est commune aux désirs et aux aspirations des jeunes postcoloniaux d'aujourd'hui. J'entends par là, des processus qui caractérisent le scénario des relations de pouvoir au niveau mondial, les valeurs qui se présentent comme hégémoniques et les formes prescrites d'être-au-monde. Dans mon analyse, je considère le désir d'assimilation de ces "formes et normes " comme un élément central de l'éthos contemporain et des subjectivités qui se définissent dans ce cadre. Avec le concept de " subjectivité " je fais référence aux modes, conscients ou non, de s'orienter au monde et d'incorporer valeurs, normes, langages et signifiants historiquement pertinents, qui influencent les représentations et l'imaginaire et que définissent des manières spécifiques de penser, d'agir, de parler, de souffrir, etc. (Ortner, 2005 ; Luhrmann, 2006 ; Biehl, Good et Kleinman, 2007 ; Moore, 2007).

Mon analyse, en outre, se fait l'écho de celle d'auteurs qui ont récemment proposé une lecture des phénomènes migratoires comme le produit des transformations sociales provoquées par le développement du marché global de nature capitaliste et par ses processus d'articulation au niveau mondial (Étatnation, expansion coloniale, idéologie du progrès et circulation transnationale). II s'agit d'un type d'analyse en ligne avec la perspective que Stephen Castles a récemment appelée "socio-transformative " et qui vise à " to embed migration research in a more general understanding of contemporary society " (Castles,

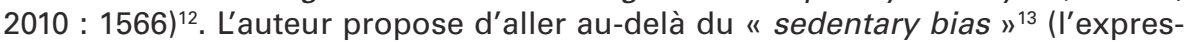
sion est de Bakewell, 2008a), qui a souvent caractérisé l'analyse des phénomènes migratoires (Castles, 2010).

En me centrant ici sur la migration des enfants " non accompagnés ", je vais tenter de discuter la matrice commune du désir migratoire de nombreux jeunes d'aujourd'hui.

12 " Insérer la recherche sur les migrations dans une compréhension plus générale de la société contemporaine " [ma traduction].

13 "Biais sédentariste" [ma traduction]. 


\title{
Formes de citoyenneté matérielle
}

\section{'Abdelhaqq}

" Dans la ville, je voyais les émigrés qui revenaient, ils avaient de belles voitures... Certains d'entre eux ont construit un palais en face de ma maison, beau, tout était là, c'était moderne... J'ai pensé que je voulais un bâtiment comme celui-là " ('Abdelhaqq, originaire de Casablanca, parti à seize ans).

\begin{abstract}
'Abdelhaqq vient de Karian Rehamna, un bidonville dans la commune de Sidi Moumen, à Casablanca. Il quitte l'école en primaire, où, dit-il, " si tu levais ta main pour une question, le professeur te chassait ". Durant les deux dernières années passées au Maroc il allait au port presque chaque jour, en essayant de " brûler " ${ }^{14}$ avec les autres enfants du quartier. Un jour, à seize ans, il a réussi à ramper sous un camion avant que celui-ci n'embarque : "Quand le navire a appareillé, nous sommes sortis tous les trois du même camion " - il rit. Après trois jours cachés dans la cale, ils ont débarqué à Marseille, où 'Abdelhaqq connaissait des gens. Il est resté chez eux pendant quelques mois, puis, lorsqu'ils lui ont proposé d'être assisté par un centre d'accueil pour les mineurs, il est parti à Turin. Des amis l'ont accueilli chez eux, mais lui ont bientôt fait comprendre qu'il devait "debber 'la rasu ", se débrouiller. La manière était facile : zetla et baida (hashish et cocaïne), " c'est ainsi qu'on fait l'argent ".
\end{abstract}

\section{Lovely}

"Une voisine est venue à la maison et a parlé avec mes parents. Elle disait qu'en Europe il y avait du travail, mais nous on savait ce que les fillettes font là-bas. On en parle beaucoup. Mais finalement qu'est-ce qu'on peut faire ? Ils m'ont amenée au "shrine", où le prêtre a fait des choses. Ils m'ont pris des cheveux et des poils, et ils ont fait des choses. Ils ont dit qu'il y avait une dame qui m'attendait en Europe et qui avait payé pour mon voyage. Je devais obéir au "connection" et je devais payer une fois arrivée. Si je ne le faisais pas, je pouvais mourir, ou quelque chose de mauvais pouvait arriver à ma famille. J'avais peur... " (Lovely, originaire de Benin City, partie à dix-sept ans).

Lovely vient de la périphérie de Benin City, capitale de l'État d'Edo, au Nigéria. En septembre 2006, après la visite d'une voisine, elle est accompagnée chez un praticien traditionnel qui organise un rituel où elle s'engage à indemniser les organisateurs de son voyage avec le produit de son travail en Europe. Lovely a peur, mais ne peut plus reculer puisqu'elle est immédiatement mise dans une voiture et emmenée loin de la famille. Après quelques jours, elle part en avion pour Accra d'où, avec un faux passeport, elle embarque avec une femme pour Amsterdam et, de là, pourTurin. Elle vit pendant deux ans avec trois autres filles de Benin City, qui tous les soirs se rendent dans une ville voisine pour se prostituer dans la rue.

Je la rencontre en 2008 dans un centre de protection pour victimes de la traite. Elle raconte que sa famille s'est trouvée en difficulté après la mort du père,

14 "Brûler " (hreg, de l'arabe haraqa) ; expression utilisée au Maroc, et au Maghreb en général, pour la migration irrégulière. A l'idée de "brûler " - les frontières, les interdictions, les documents pour ne pas être rapatriés - s'ajoute souvent celle de " risquer" (risker). Pour une discussion de ces métaphores, voir Pandolfo (2007) ; Vacchiano (2010). 
qui aurait laissé ses biens à sa première femme. Elle ne voulait pas partir, mais la famille l'avait convaincue en lui disant qu'elle obtiendrait ainsi de l'argent et que plus tard, après quelques années, elle reviendrait à la maison aussi riche que ces autres femmes qui avaient fait fortune en Europe. Entre-temps, elle pouvait envoyer de l'argent pour payer l'école de ses frères.

\section{Safdar}

"Au Pakistan, dans la famille où je vivais, j'étais bien traité mais la vie était difficile. Les Afghans sans papiers ne peuvent pas aller à l'école et beaucoup de bons gars, après un peu de temps, partent pour aller en Iran ou même au-delà. Beaucoup avaient déjà quitté pour aller en Europe... Je pensais que je pouvais étudier en Europe, être un peu plus tranquille et vivre un peu mieux, avec les choses que me manquaient là-bas "

(Safdar, originaire de Ghazni, parti à neuf ans).

Safdar est un jeune hazara, minorité de religion chiite fixée depuis des siècles dans la région centrale de l'Afghanistan. En 1999, à neuf ans, il quitte son village pour la première fois afin d'éviter le recrutement forcé mis en place par les talibans de sa province. Dans la ville de Quetta, au Pakistan, il vit avec d'autres réfugiés, sans famille et sans documents. Après moins d'un an, avec l'accord de sa mère, il se joint à des compatriotes pour tenter de rejoindre l'Australie, mais le groupe est arrêté et détenu dans un centre de détention en Indonésie. L'enfant y reste pendant sept mois, après quoi il est rapatrié. À son retour, sa famille n'est plus dans son village. Safdar se cache chez un ami et, après quelques mois, décide de rentrer au Pakistan, où il trouve hospitalité chez une famille pakistanaise, en échange de son travail en tant que vendeur dans leur entrepôt. Pendant son temps libre, il étudie l'anglais, qu'il utilise pour chercher sa famille sur Internet.

"Je suis alors entré en contact avec des amis qui vivaient en Europe. Je voulais étudier et être en mesure de vivre une vie normale, trouver un travail et retrouver ma famille. J'ai pensé que je devais aller et faire comme eux".

Après encore deux ans d'attente, Safdar part pour un autre voyage, cette fois-ci en direction de l'Europe, à travers I'Iran et la Turquie, pour rejoindre la Grèce. À chacune de ces étapes, il assiste - ou est personnellement victime - à des violences et abus de la part des trafiquants et de la police. Puis il part de Grèce, et après plus de cinquante heures caché dans un véhicule de transport, il atteint la frontière italienne. Suite à un bref passage à Rome, il arrive àTurin où avait-il appris - vivait un jeune homme de son village. L'ami le met en contact avec les services sociaux, qui le placent alors dans une famille d'accueil. Quand je I'ai connu, en 2006, Safdar attendait la réponse à sa demande d'asile, avec beaucoup d'anxiété et d'espoir.

Qu'ont en commun des histoires aussi différentes que celles de 'Abdelhaqq, Lovely et Safdar ? À première vue, uniquement le fait qu'elles concernent des enfants ou adolescents qui, pour des raisons très différentes, quittent leur pays et entament le long et dangereux chemin de la migration internationale. Certains d'entre eux partent de leur propre gré, d'autres pour exaucer les désirs de leur famille, d'autres encore contraints par des conditions d'insécurité, d'autres enfin sont forcés ou enlevés. Leurs histoires laissent voir une pluralité de situations et de parcours qui montrent que la migration autonome des mineurs a des raisons 
complexes et des dynamiques très différentes : problèmes économiques, malaise social, traite et exploitation, sujétion ou besoin d'échapper à l'insécurité et à la violence. Cependant, un élément transversal renvoie à une série de modèles d'existence considérés comme souhaitables en opposition à ceux expérimentés, et expérimentables, dans son propre contexte de vie. Dans les mots avec lesquels les jeunes parlent de leur projet migratoire, ces modèles apparaissent sous la forme de standards de vie désirables avec lesquels la comparaison se fait constamment. Que le voyage soit considéré comme un choix ou qu'il se fasse par la volonté d'autrui - famille, réseaux de trafic, ou pour d'autres raisons de force majeure -, le faisceau de valeurs globales qui définissent les formes de vie jugées souhaitables représente l'arrière-plan des processus de mobilité et influence en profondeur ses motifs. II ne s'agit évidemment pas de sous-estimer l'importance des facteurs tels que l'exclusion, la marginalité, le risque, la guerre ou la violence dans toutes les acceptions possibles, ni l'acuité des problèmes sociaux, qui dans certains contextes peuvent encourager les enfants et les adolescents à partir. Néanmoins, je propose de centrer l'analyse sur les liens entre la mobilité et ce que nous pourrions appeler un " éthos contemporain des opportunités".

La majorité des jeunes qui migrent seuls vient de quartiers populaires ou de banlieues où grandir s'accompagne d'une prise de conscience de l'exclusion et de l'absence de toutes possibilités de promotion sociale. Pour les jeunes qui ont connu la guerre (Afghanistan, Syrie, Somalie), le voyage, en dépit de ses difficultés bien connues, est souvent une façon de se protéger (pour qui part, mais aussi pour qui reste et qui investit sur une aide future pour fuir à son tour). Malgré ça, l'importance de ces facteurs structurels doit être évaluée en relation étroite avec le scénario global contemporain, dans lequel les enfants grandissent plongés dans un " écoumène " (Hannerz, 1996) fait d'images, de représentations et de valeurs largement partagées. Le cas des mineurs qui se retrouvent dans les réseaux de traite montre bien souvent que ces jeunes, recrutés dans des situations de précarité personnelle ou familiale, sont attirés par les promesses d'une vie considérée comme "plus digne " selon des modèles d'existence et d'expérience perçus comme " plus modernes". Ces modèles s'appuient sur un " autre en soi " déjà bien présent et configurent un " ailleurs imaginaire " où se manifestent les signes d'un statut différent.

"Ils m'ont dit que je pouvais travailler comme vendeuse dans un magasin, ou peut-être en tant que commise... que j'aurais ce que je voulais... J'y croyais parce que j'avais vu des gens qui revenaient bien habillés, avec des voitures, et je voulais être comme eux " (Elena, originaire de Bucarest, partie à dix-sept ans).

"Au centre de Casablanca, il y a plein de gens bien habillés. Tu les regardes et tu as déjà honte... J'y allais pour les regarder et je pensais que je voulais des chaussures, une voiture. Elles étaient trop belles. Si je vais au Marjan [supermarché], le gardien me renvoie. Il sait que je n'ai pas d'argent et je finis par faire du bordel " (Sa'id, originaire de

Casablanca).

Le "palais moderne " d'Abdelhaqq, son désir des " choses normales ", les maisons et l'argent souhaités par la famille de Lovely, "les choses qui manquaient " et le désir légitime de vivre " normalement " de Safdar, en plus des promesses qu'Elena feint de croire et le bien-être à la fois proche et interdit 
de Sa'id, mettent en évidence l'impact violent des figures " modernes " du succès sur ceux qui en sont exclus.

À travers ces figures de la privation et du désir, on distingue les valeurs fondamentales de l'être-au-monde contemporain, qui imprègnent la plupart des contextes d'où les jeunes proviennent. II s'agit de " modèles " qui relient le statut de l'individu à sa capacité d'acquisition et de consommation, aux possibilités intrinsèques du mouvement et de la communication permanente, à l'autonomie individuelle et à la subversion de l'ordre des générations. Leur impact est particulièrement important pour la définition de la subjectivité de la jeunesse dans le monde contemporain et pour la construction imaginaire sur laquelle elle s'appuie (Comaroff et Comaroff, 2005). Comme I'observe Fass, " adolescence may have become a universal identity in the context of globalization as the accoutrements and culture of youth have spread everywhere through the very channels that define globalization "(Fass, $2005: 348-349)^{15}$.

La consommation a une place particulière dans ce scénario : ses dynamiques concernent le statut des sujets et la définition sociale de leurs capacités (Miller, 2012). Les auteurs qui ont travaillé sur la compréhension de la consommation ont largement mis en évidence la dimension éminemment sociale des objets, ainsi que les aspects liés à leurs différents " contextes d'utilisation " (Appadurai, 2009 ; Howes, 1996). Dans un texte de 2003, Aldridge mettait en relation consommation, insécurité et crise du lien social, notant que la consommation devient un champ d'investissement compensatoire dans lequel se négocient, identités, sentiment d'appartenance et accomplissement de soi (Aldridge, 2003). Parmi les jeunes migrants et adolescents avec lesquels j'ai travaillé, le désir d'une vie meilleure était souvent exprimé à travers un vocabulaire spécifique des choses, qui révélait leur valeur en tant qu'objets de " confort " (Miller, 2008). La voiture en est un exemple, qui traduit à la fois l'évolution sociale rapide et le mouvement inhérent au statut " moderne " du sujet.

La consommation dans ses formes visibles devient ainsi un puissant moyen de participation au monde, tant en termes matériels que sur un plan symbolique, par la possibilité d'être " modernes" dans un sens moral (Bauman, 2007). Comme beaucoup de jeunes en témoignent, c'est souvent sous forme d'inégalité par rapport aux possibilités d'acquisition que le sentiment d'isolement et d'immobilité se définit : les " choses normales " (et normatives) - c'est-à-dire les réalisations matérielles qui contribuent à rendre la vie acceptable aujourd'hui sont dépeintes comme gardées artificiellement à distance, souvent même comme le montre le témoignage de Sa'id - dans un sens spatial. Il s'agit, comme Mbembe l'a observé, d'une "économie des désirs inassouvis ", liée à des " biens dont on a connaissance; que parfois I'on voit ; que l'on désire posséder ; dont on veut jouir, mais auxquels I'on n'aura jamais accès " (Mbembe, 2000 : 41). Ces biens "emblématiques ", qui représentent le succès et la mobilité (et aussi le succès de la mobilité) reviennent au sujet sous forme de " dépossession " (I'expression est de Stearns, 2006), en créant une dynamique de soustraction dans laquelle les jeunes et leurs familles se retrouvent aux marges d'une

15 "L'adolescence devient une identité universelle dans le contexte de la mondialisation en même temps que les accessoires et la culture juvénile se répandent partout par les mêmes canaux qui constituent la globalisation " [ma traduction]. 
citoyenneté matérielle qui représente en même temps la base concrète de leur être et le fondement de leurs expectatives de bien-être.

\section{La mobilité comme succès}

Dans ce processus de comparaison subjective avec les objets, la mobilité apparait comme le principal vecteur de possibilités, comme les "success stories " des émigrants viennent souvent le montrer. Dans les discours de nombreux jeunes, les visites des émigrants représentent une sorte de "choc " du fait du contraste entre leur bonheur immédiat - forcément ostentatoire - et la perception d'immobilité qui accompagne le quotidien de ceux qui restent. Le sentiment d'emprisonnement est d'autant plus fort qu'il contraste avec la liberté de ceux qui ont le droit de se déplacer. L'image sélective des " migrants chanceux " est par ailleurs associée à d'autres figures qui incarnent le pouvoir différentiel du mouvement : les élites du pays, les touristes, les hommes d'affaires, les sportifs, etc. À partir de cet imaginaire de liberté et des possibilités qui y sont associées, la mobilité se charge d'une valeur relativement autonome : si d'une part le désir de partir mûrit dans des conditions sociales et structurelles spécifiques, celles-là même sont d'autre part de plus en plus interprétées en relation avec les possibilités de mouvement. II est manifeste aujourd'hui que ceux qui détiennent le pouvoir (économique, politique, social) ont accès à différentes formes de mouvement et que, dans de nombreux cas, leur mobilité est aussi l'outil principal de reproduction de leur pouvoir social. Le cas le plus marquant est celui des enfants des classes dirigeantes de pays comme le Maroc, le Nigéria, la Tunisie, le Sénégal, etc., formés principalement dans des établissements privés ou étrangers et destinés à des études supérieures dans des universités américaines ou européennes. Leur cosmopolitisme leur confère un avantage sélectif vis-à-vis de leurs pairs et les prédestine à occuper, à leur retour, des rôles de leadership dans les domaines des affaires, de la politique, de I'université, de la communication et de l'administration de l'État.

Le pouvoir différentiel entre ceux qui peuvent se déplacer et ceux qui sont " confinés " est tout simplement incommensurable, parce qu'il est surtout activement maintenu par des stratégies sélectives construites sur la gestion de la mobilité. Nombreux sont les auteurs qui ont mis l'accent sur cet aspect et qui ont identifié dans les mécanismes du "régime des frontières " contemporains un outil de sélection hautement discriminatoire. Une discussion spécifique de ce sujet dépasse l'ambition de ce travail, mais il reste important de noter ici que les formes de contrôle de l'immigration mises en place par l'Europe, en raidissant les frontières et en renforçant leur surveillance, opèrent en synergie avec les forces conservatives qui dans les pays d'origine maintiennent la stratification sociale héritée de l'époque coloniale et postcoloniale. On peut comprendre alors que les jeunes aspirent au mouvement et au changement comme manière intrinsèque de participer au monde et comme possibilité $d^{\prime}$ " être dans le temps".

D'une façon générale, l'importance de la mobilité pour les nouvelles formes de pouvoir et de statut dans le monde globalisé avait déjà été détectée par Bauman en 1998: "Mobility climbs to rank of the uppermost among the coveted values - and freedom of movement, perpetually a scarce and unequally distributed commodity, fast becomes the main stratifying factor of our late-modern 
or postmodern times. [...] Being local in a globalized world is a sign of social deprivation and degradation" (Bauman, $1998: 2)^{16}$.

Dans un monde en mouvement (Bauman, 2000 ; Sheller et Urry, 2006 ; Urry, 2007 ; Salazar et Smart, 2011 ; Glick Schiller et Salazar, 2013), I'immobilisation est ressentie comme l'une des injustices les plus profondes. On peut dire que le " droit de se déplacer ", avec le " droit de consommer ", sont parmi les principales attentes qui façonnent les formes contemporaines d'être-au-monde, en particulier chez les jeunes. Concernant ces " dispositions morales incorporées ", la migration vers l'Europe est perçue comme un acte compensatoire d'affirmation de soi ainsi qu'une façon de participer au monde en saisissant ses opportunités de réussite matérielle. De cette manière, le départ "à tout prix " est souvent entendu - et réclamé - comme un défi à un ordre social injuste, bien qu'exprimé à travers le lexique privé de la réussite matérielle et du succès personnel.

La condamnation à " être local " - immobile, écarté, isolé dans les marges d'un espace aliéné - est vécue par les jeunes comme une souffrance inacceptable. Seule la communication par Internet - une forme de mobilité " imaginée " vient parfois l'atténuer. Beaucoup d'adolescents, sans distinction d'origine et de façon très semblable à leurs pairs dans le monde, passent beaucoup de temps dans les cybercafés en essayant de surmonter les distances et de reconstruire, à travers la communication (" rendre commun ») un sentiment d'appartenance à une communauté plus vaste. Grâce à la toile, les jeunes s'ouvrent sur le monde et nourrissent un " imaginaire de fugue " à travers les contacts avec des amis, des membres de la famille et des connaissances, réels et virtuels. Dans les réseaux sociaux, ils partagent des photos, de la musique, des messages, et surtout leurs expériences avec les amis qui ont émigré, se renseignant sur les centres d'accueil en Europe, les routes pour échapper aux contrôles, les stratégies de survie quotidienne et les moyens de franchir les frontières. Ils glanent aussi des informations utiles sur la vie quotidienne et apprennent des langues étrangères. Grâce à la toile, ils alimentent tout un imaginaire proprement translocal - un " horizon global " (Graw et Schielke, 2013) de valeurs et d'aspirations projeté dans un " ailleurs " anticipé et désiré ; comme ce garçon de quinze ans rencontré à Fès en 2006, qui passait sa journée virtuellement projeté en Angleterre, étudiant la langue, suivant l'actualité, communiquant par chat avec les personnes qu'il rêvait de rencontrer.

$L^{\prime}$ « ailleurs " est le lieu des possibles et des alternatives, du mouvement et de la liberté, où l'individu pourra enfin trouver un espace d'expression et une réparation de l'injustice. Ce n'est pas seulement un lieu géographique (parfois il n'est pas précisé), mais une entité métaphysique, définie par des projections de soi et de la réalité. C'est un lieu imaginaire construit sur l'idée d'une compensation symbolique qui prend une forme matérielle dans les signes d'une modernité disponible pour ceux qui sont en situation d'en profiter. Un " exil hors d'exil ", comme Fouquet le définit par rapport à l'expérience des jeunes dakarois : " un

16 « La mobilité prend le niveau le plus élevé parmi les valeurs qui confèrent un statut, et la liberté de mouvement - toujours un bien rare et inégalement réparti - devient rapidement le principal facteur de stratification sociale de notre temps. [...] Être local dans un monde globalisé est un signe d'infériorité et de dégradation sociale " [ma traduction]. 
espace d'imaginaires dépositaire des aspirations à un mieux être et à un mieux vivre " (Fouquet, 2007 : 84). Le présent est progressivement investi par la force de ses images et la quête de sens pour le quotidien aboutit sur une certitude : l'" ailleurs " est le seul lieu où la vie mérite d'être vécue. Le risque ne semble plus si effrayant et une rhétorique du sacrifice pour les autres - la famille d'abord - compense la possibilité de " se perdre " dans ses séductions. Même la mort ne peut plus faire peur à qui, au fond, vit l'immobilité comme la forme la plus douloureuse de " mort en vie".

\section{"Sauver les parents"}

De nombreux travaux montrent qu'en différents contextes les enfants représentent une ressource "naturelle " pour la survie de la famille, et ce, pas uniquement dans les sociétés dites "traditionnelles". Dans les milieux agricoles, mais aussi dans les classes populaires, les enfants et les adolescents contribuent à l'économie du groupe, particulièrement en période de crise économique. Ainsi la mobilité joue un rôle significatif dans ce processus de participation aux ressources familiales. On connait bien le cas des enfants italiens qui, au $\mathrm{XIXe}$ siècle, jouaient de I'orgue dans les rues françaises, ainsi que celui des enfants et des adolescents envoyés en "service domestique ", sous différentes formes et dans différents endroits du monde, pour contribuer aux ressources de leurs familles ou simplement alléger la charge de leur entretien. Les exemples sont nombreux : les enfants enlevés à Londres et envoyés en " service domestique " dans le nouveau monde (Fass, 2005) ou le cas du "Fairbridge Scheme ", un programme parrainé par la Society for the Furtherance of Child Emigration to the Colonies et conçu pour envoyer les enfants orphelins ou de familles pauvres du Royaume-Uni vers des "fermes pédagogiques " de l'empire (Gill, 1998 ; Jeffery et Sherington, 1998). Comme l'observe Fass, "that leap in our imagination of what children ought and ought not to do is the product of Western history and of the development of humanistic sentiments during the last two hundred years " (Fass, $2005:$ 939) ${ }^{17}$.

Paul Stearns, alors qu'il s'intéresse au paradigme de l'enfance, montre comment le changement de représentation des enfants en Europe et en Amérique du Nord a été influencé par les idées des Lumières et du Romantisme. Durant la période d'industrialisation, la scolarisation progressive, le contrôle des naissances et la baisse de la mortalité infantile modifient significativement le statut de l'enfance et les rapports entre les parents et leurs enfants. Cependant, cette transformation, qui touche principalement l'Europe du Nord et les ÉtatsUnis, n'est pas observée de la même manière dans toutes les régions du monde. Alors que l'internationalisation de l'idée d'enfance en tant que catégorie vulnérable a été activement promue (par exemple par des institutions telles que I'UNICEF), I'accent mis sur la primauté du marché et des privatisations (défendu par d'autres organisations comme le FMI) a limité la capacité des États à libérer les enfants des tâches productives au bénéfice de leurs familles (Stearns, 2005).

17 « Le saut dans notre imaginaire entre ce que les enfants devraient et ne devraient pas faire est le produit de I'histoire occidentale et du développement d'une sensibilité humaniste au cours des deux cents dernières années " [ma traduction]. 
Dans de nombreux pays, les familles "prolétaires " (étymologiquement, dont le seul patrimoine est la descendance) ont encore besoin de leurs enfants pour survivre. Dans cette logique, le scénario des opportunités globales offre des possibilités renouvelées. Pour Fass, " even in the most traditional and hierarchical societies and during periods of significant change, children may in fact be seen as the salvation of the family" (Fass, $2005: 949)^{18}$. Et c'est bien ce que disent, presque mot pour mot, certains jeunes Marocains qui justifient leur entreprise migratoire comme la possibilité de "sauver leurs parents " ('ataq I-wālidìn) (Vacchiano, 2010).

" Je suis venu pour sauver mes parents... Ils sont pauvres et ne peuvent rien faire. Mon père est à la maison, "couché" [sans-emploi]... mon frère a un problème mental, mais il n'y a pas d'argent pour les médicaments... Un autre frère est vendeur et gagne un salaire de misère... Au Maroc, de temps en temps je vendais des sacs en plastique au marché, mais je veux faire d'autres choses dans la vie " (Kamal, originaire de Casablanca,

dix-sept ans).

"Ma mère est en Iran avec mon plus jeune frère. J'ai pu lui parler à travers quelques connaissances qui m'ont donné le numéro d'autres amis... Je veux travailler pour être en mesure de les emmener ici. La vie est dure là-bas et les Afghans sont maltraités. C'est la chose que je désire le plus dans ma vie. Après je pourrais penser à moi-même " (Salim, originaire de Kandahar, seize ans).

Ainsi de nombreux adolescents migrants font référence à la solidarité familiale et à la responsabilité envers ceux qui sont restés pour donner sens à leur projet transnational. Le lien avec la famille d'origine et les obligations qui en découlent restent importants dans le cas de la migration autonome. Ce n'est pas seulement parce que l'expérience de la migration est mesurée en fonction du contexte d'origine et du groupe qui la ratifie, mais aussi parce que la référence à la famille à " sauver " justifie, à travers une rhétorique socialement acceptée, un projet dont l'issue est toujours potentiellement ambiguë. L'investissement des familles dans la migration de leurs enfants est aussi un pari sur le maintien du lien familial, souvent déjà en crise en raison de conflits politiques, des guerres, des restructurations économiques et, surtout, à cause des changements provoqués par les transformations sociales qui caractérisent les contextes d'origine. L'impact de ces facteurs sur les structures locales est considérable et met en tension sinon les hiérarchies, du moins les rôles sociaux en matière de production et de reproduction. Comme le souligne Suárez-Navaz: " El impacto de las relaciones de producción capitalistas en la transformación de las relaciones de género y generación ha sido documentado contundentemente a lo largo y ancho del mundo. La proletarización de jóvenes adultos, mujeres y menores trastoca en su esencia el sistema de dependencia en el que se basaba no sólo la producción doméstica, sino la autoridad política y los principales

18 " Même dans la société la plus traditionnelle et hiérarchique, et en période de changements importants, les enfants peuvent être considérés comme le salut de la famille " [ma traduction]. 
mecanismos de cohesión social » (Suárez-Navaz, $2006: 6)^{19}$.

La crise prend forme dans l'épuisement des figures d'autorité traditionnelles (les pères en premier lieu), dans leur expulsion du marché du travail et dans la perte progressive de leur capacité de transmettre aux enfants une lecture plausible du monde et de sa complexité. Elles sont autant affirmées publiquement que concrètement dévalorisées dans le quotidien où les enfants ont toute latitude pour constater leur échec historique (Vacchiano, 2010). Dans ce cadre, qui combine l'érosion des figures d'autorité, la crise des formes de reproduction sociale et l'hégémonie croissante des valeurs globales, les enfants et les jeunes deviennent les meilleurs interprètes d'un changement duquel les adultes semblent déjà exclus. Leur responsabilité s'inscrit dans un univers de possibilités plus large qui est représentatif de la géographie contemporaine des mouvements considérés comme envisageables, grâce aussi à l'appui des réseaux diasporiques transnationaux.

Dans l'articulation complexe de cet "espace transnational des opportunités ", les enfants se définissent en tant que sujets éminemment productifs : ils accomplissent une mission de transformation pour la rédemption sociale et historique des parents et du groupe. Dans certains cas, comme le prouve le témoignage de Salim, le "sauvetage des pères " est loin d'être seulement symbolique.

La tendance des mineurs à remplacer les adultes peut être rapportée, dans certains cas, à un effet sélectif des politiques migratoires européennes, qui, en prescrivant une différence de statut pour certains sujets "vulnérables ", créent, au moins formellement, un différentiel par rapport à la " déportabilité " (De Genova, 2002) des adultes. Ma recherche de terrain montre que, dans certains cas, des familles choisissaient d'investir sur le départ de leurs enfants car elles imaginent parfois les centres d'accueil en Europe comme pouvant les remplacer efficacement ou comme un instrument de welfare transnational. Néanmoins, il s'agit d'un trop petit nombre de cas pour attribuer aux politiques de protection - par ailleurs prévues par le droit international - la responsabilité d'un hypothétique " effet d'appel ", comme certaines administrations publiques I'ont fait (Vacchiano et Jiménez, 2012). On a vérifié sur le terrain, en revanche, un bon nombre de situations où les familles essayaient d'éviter l'intégration de leurs enfants dans les structures d'accueil, dans l'attente d'un bénéfice économique immédiat, avant qu'ils ne deviennent majeurs et, donc, expulsables.

De toute façon, les enfants arrivés en Europe au cours des dernières années expriment un projet personnel ou familial qui transcende largement les limites imposées par les politiques migratoires ou par les systèmes d'accueil de l'enfance migrante. La force de cette propension à la mobilité, qui dépasse et défie les limites de l'ordre postcolonial et néocolonial doit être pensée principalement comme le résultat d'une nouvelle " conscience du monde " - une " sensibilité globale "- dont les jeunes sont porteurs et à partir de laquelle

19 « L'impact des formes de production capitaliste sur la transformation des relations entre sexes et entre générations est profondément documenté dans le monde entier. La prolétarisation des jeunes adultes, des femmes et des enfants, modifie considérablement le système de dépendance sur lequel s'appuyait non seulement la production domestique, mais aussi l'autorité politique et les principaux mécanismes de cohésion sociale " [ma traduction]. 
la " crise des pères " peut, à son tour, être comprise. Les jeunes entérinent la " défaite des pères " devant les exigences d'un monde " moderne " dont ils se considèrent comme les interprètes légitimes. En même temps, les parents socialement perdants des classes inférieures confient à leurs enfants leurs attentes pour une rédemption dont le sens, sans doute existentiel, se définit selon des formes éminemment matérielles.

\section{" Être modernes " : un problème de citoyenneté}

Dans un article publié dans la revue Cultural Anthropology en 2002, I'anthropologue américain James Ferguson analysait la fameuse lettre de Yaguine Koita et FodéTounkara, deux garçons âgés de quatorze et quinze ans retrouvés morts, en août 1998, à l'aéroport de Bruxelles dans le train d'atterrissage d'un avion Sabena en provenance de Conakry (Ferguson, 2002). Le texte, très connu, a été également utilisé dans des publications sur la migration de mineurs pour montrer la force et les raisons de leurs objectifs. La lettre est adressée aux "Excellences, Messieurs les Membres et Responsables d'Europe " auxquels " nous, les enfants et jeunes d'Afrique " demandent de l'aide - au nom de "I'amour de votre continent, pour le sentiment que vous avez envers votre peuple et surtout pour l'affinité et l'amour que vous avez pour vos enfants que vous aimez pour la vie " - afin de " faire une grande organisation efficace pour I'Afrique pour nous permettre de progresser ". En particulier, les deux jeunes demandent une solution aux " guerres, maladie, manque de nourriture [...] manque d'éducation et d'enseignement ", mais aussi pour le manque " d'écoles sportives où [...] pratiquer le football, le basket ou le tennis ". La lettre fait référence au désir d'étudier, un moyen de progresser et de s'améliorer, à travers une demande qui ne peut pas passer inaperçue : " nous vous demandons de nous aider à étudier pour être comme vous en Afrique ".

Le message, très émouvant par le contenu et les circonstances de sa découverte, frappe par l'analyse que les deux garçons font de la discrimination sociale dans leur pays - "sauf dans les écoles privées [...] I'on peut avoir une bonne éducation et un bon enseignement, mais il faut une forte somme d'argent " et pour la référence aux sources de cette inégalité : " n'oubliez pas que c'est à vous que nous devons nous plaindre de la faiblesse de notre force en Afrique ". L'analyse de Ferguson s'appuie sur son - et notre - personnel " embarrassment of encountering Africans - in this ostensibly postcolonial era - who humbly beg Europeans to come to their aid and who bluntly ask for help "to become like you" "(Ferguson $2002: 552)^{20}$. D'autre part, reconnaît Ferguson, cet embarras n'est pas trop différent de la sensation ressentie par de nombreux anthropologues sur le terrain en Afrique quand leurs interlocuteurs les mettent devant des " nostalgic reminiscences of colonial days or passionate appeals for salvation from Africa's problems via some imagined "return" of whites who might "help us to become like you" "(Ferguson $2002: 552)^{21}$.

20 « Embarras à trouver des Africains - dans notre époque apparemment postcoloniale qui demandent humblement aux Européens d'aller à leur secours et qui demandent ouvertement de I'aide à "être comme vous" " [ma traduction].

21 "Réminiscences nostalgiques des jours coloniaux ou des appels passionnés pour le salut de I'Afrique à travers l'imaginaire "retour" des Blancs qui aident à "devenir comme vous" " [ma traduction]. 
James Ferguson analyse la relation entre " mimesis" (le " devenir comme vous ") et le désir d'appartenance " à une société plus large " dans le débat anthropologique sur quelques cas célèbres $d^{\prime}$ " imitation " des Blancs durant la colonisation en Afrique. Il démontre que les analyses qui ont tenté de donner une dignité " de résistance " à la conduite des sujets coloniaux ont souvent perdu de vue l'objet spécifique des comportements examinés, c'est-à-dire l'émulation en soi, sans doute explicable comme produit de la situation coloniale, mais aussi en tant que désir d'appartenance à un monde plus vaste et volonté $\mathrm{d}^{\prime}$ " inscription dans la société mondiale " (Ferguson, $\left.2002: 558\right)$. Le fait que cela soit dû à l'efficacité de la construction hégémonique coloniale, à son tour liée aux relations historiques de pouvoir, n'en réduit pas l'importance du point de vue subjectif.

La lettre évoque la compréhension, mais aussi la "solidarité et gentillesse " des dirigeants européens pour construire une " grande organisation efficace ", qui " permet de progresser " vers un modèle de vie perçu comme juste, dans lequel les jeunes peuvent vivre sans guerre, manger, aller à l'école et faire du sport : le modèle pour lequel ils sont disposés - comme le soutiennent Yaguine et Fodé - "à faire des sacrifices et mettre leur vie en danger ". Si, comme I'on entend parfois dire " je n'ai pas peur de mourir, la mort est ici ", c'est parce que l'investissement quotidien dans son propre pays est vu simplement comme vain et sans succès. En ce sens, la " migration à tout prix " n'est pas un acte désespéré, mais la tentative de se connecter au flux du monde, au rythme des choses, à l'être-au-temps contemporain, au statut d'un sujet imaginé comme " moderne $"^{22}$.

Comme le propose Ferguson dans ses travaux, à l'époque des « nations émergentes " la rhétorique du développement ancrait la perspective de la modernité dans l'idée d'un progrès possible et souhaitable pour tous, à condition de suivre le chemin principal de l'industrialisation qui va main dans la main avec la science et la technologie, les politiques libéro-démocratiques, la famille nucléaire et la vision sécularisée du monde. Dans cette vision, la modernité apparaît comme un grand "telos universel ", dans lequel toutes les sociétés, avec du temps et de la patience, arriveraient à atteindre le stade souhaité du développement. Malgré ses promesses, ce récit rangeait les sociétés " en développement " dans l'ombre de celles qui ouvraient le chemin et préparait les interventions pour " corriger le cap " (privatisations, ajustements structurels, libéralisation du marché, etc.), en direction d'un succès reporté sans cesse.

"On n'a plus de patience ", comme le disent aujourd'hui des jeunes migrants aux frontières de l'Europe : si le temps du développement était un temps diachronique d'attente et d'espoir - un " pas encore " projeté dans l'avenir celui de la globalisation est le temps synchronique du " tout et tout-de-suite ", où la vitesse impose la nécessité de saisir les opportunités et de choisir rapidement, sous peine d'exclusion irréversible du flux des changements. "Être moderne " aujourd'hui ne se termine pas dans la perspective de l'attente, dans un telos renvoyé pour un avenir possible, mais s'exprime dans la nécessité d'assumer le

22 J'utilise le concept de modernité dans son sens étymologique : le concept de modo, qui rappelle un temps (en latin il est " naguère " ou " peu après ") et une manière, spécifique, de faire les choses. Voir aussi Fergusson, 2002 et 2006. 
changement dans le moment historique où il se présente, de l'intégrer en incorporant ses formes et son rythme, d'acquérir ses qualités en vue d'un nouveau statut : "Modernity in this historically specific conjuncture appears [...] as a global status and a political-economic condition: the condition of being "fist class" "23 (Ferguson, 2006 : 187).

C'est cette condition, fondée sur le succès visible d'une forme de vie historiquement gagnante, qui rend plausible une "éthique des possibilités " dans laquelle le sujet se projette et se définit au-delà de l'espace quotidien, au-delà des limites sociales, dans des lieux de l'imaginaire où il est possible d'être soi-même et autre en même temps. Si, comme l'a noté Benslama, la modernité incarne "le désir d'être autre "(Benslama, 2004), c'est également parce que les figures d'un monde " de première classe " se sont structurées au fil du temps comme le modèle souhaitable d'altérité, auquel I'on participe à travers le mimétisme, qui est toujours aussi une forme d'appropriation. En ce sens, nous pourrions répondre à la question que Ferguson pose dans son article de 2002 - "What becomes of mimicry in a transnational, postcolonial age ? "24 notant que cette dernière prend forme aujourd'hui dans le désir d'appartenir à une société mondiale par le moyen du droit à " être comme ", acquis surtout à travers les possibilités de l' "être ailleurs".

Demander aux Européens de " nous aider à devenir comme vous" - observe encore Ferguson - " is neither a mocking parody nor a pathetically colonized aping but a haunting claim for equal rights of membership in a spectacularly unequal global society [...] ] moral claim to something like global citizenship " (Ferguson, $2002: 565)^{25}$. Pour beaucoup de jeunes postcoloniaux, cette demande ne peut être exprimée que par l'exercice d'un " droit de fuite " (Mezzadra, 2001), dans des itinéraires migratoires souvent risqués qui démontrent que I'« être global " dans le monde d'aujourd'hui est à la fois la forme la plus plausible d'être-au-monde et le privilège sur lequel des nouveaux modes d'exploitation et d'exclusion s'appuient.

\section{Conclusion}

Le paysage des mobilités humaines contemporaines actualise dans des formes renouvelées - et, selon certains, plus intenses - des processus de longue haleine (Castles et Miller, 2009 ; Palidda, 2008). En ce sens, chaque phénomène doit être lu dans la continuité de la longue durée et dans ses reformulations successives qui le reproduisent et le transforment en en faisant un phénomène ancien et nouveau à la fois. Les enfants et les adolescents ont toujours été les interprètes, et souvent les protagonistes, des mouvements migratoires. Mais

23 « La modernité dans sa conjoncture historique spécifique émerge [...] comme status global et condition politico-économique : la condition d'être "première classe" " [ma traduction].

24 «Qu'advient-il de la mimesis à l'époque postcoloniale et transnationale ? " [ma traduction].

25 « Ce n'est ni une parodie moqueuse ni une pathétique farce coloniale, mais la réclamation insistante du même droit d'appartenance à une société mondiale extraordinairement inégale [...] une revendication morale à quelque chose qui ressemble à une citoyenneté globale " [ma traduction]. 
on pourrait aussi reconnaître à quel point ce rôle de premier plan a été rendu opaque à cause de la nouvelle construction de l'enfance, un processus qui a commencé avec la révolution industrielle et s'est concrétisé dans le projet de la société libérale de l'après-guerre (Ariès, 1960 ; Becchi et Julia, 1996 ; Heywood, 2001). Cependant, I'analyse des discours et des motivations des acteurs d'aujourd'hui met en évidence la relation de ce phénomène avec un " esprit du temps " relativement nouveau. Si les mobilités humaines ont toujours été liées à la volonté de leurs acteurs d'améliorer leur condition, les dimensions de cette " amélioration ", dans le paysage global actuel, expliquent de manière hautement emblématique la structure des valeurs hégémoniques, les formes normatives de l'être-au-monde contemporain et les nouvelles configurations du pouvoir - et par conséquent, de sa privation. La reconnaissance de cet aspect est utile non seulement pour une lecture générale des contours de l'époque actuelle, mais aussi pour comprendre la condition historique des jeunes et de leurs familles dans les pays du Sud global, exposés à l'influence de ces modèles et en même temps évincés de la possibilité de se les approprier en les ré-élaborant.

Dans cet article, j'ai tenté de montrer que les jeunes migrants sont des sujets " modernes " puisqu'ils sont subjectivement construits sur les impératifs du modo contemporain, qui définit les objectifs de développement individuel et collectif à partir de formes de status basées sur la consommation, la mobilité et l'individualité en tant qu'outils et valeurs. Leur immobilisation - et celle de leurs familles -, en reproduisant les hiérarchies de classes, met en évidence le pouvoir différentiel de ceux qui peuvent se déplacer (les émigrants, les touristes, les élites nationales, les sportifs) et renforce le désir d' " être comme eux " grâce à la mobilité.

Dans la plupart des cas rencontrés, la migration " à tout prix " est interprétée comme une exigence et un défi à un ordre social injuste et oppressif, une revendication d'appartenance et une demande de " citoyenneté globale " exprimée à travers le vocabulaire normatif de l'acquisition et du succès. C'est l'affirmation d'un cosmopolitisme matériel qui, venant d'en bas, libère et asservit en même temps : il libère car il permet de briser les limites socialement imposées, il asservit car il prédispose ses acteurs à des formes de contrôle et d'exploitation que la frontière, et ses projections multiples dans le quotidien, encore une fois, leur imposent. 


\section{Références bibliographiques}

Appadurai Arjun (2009) The social life of things: commodities in cultural perspective, Cambridge, Cambridge University Press, 329 p.

Ariès Philippe (1960) L'enfant et la vie familiale sous I'ancien régime, Paris, Plon, $316 \mathrm{p}$.

Bakewell Oliver (2008a) "Keeping Them in Their Place": the ambivalent relationship between development and migration in Africa, Third World Quarterly, 29 (7), pp. 1341-1358.

Bakewell Oliver (2008b) Research Beyond the Categories: The Importance of Policy Irrelevant Research into Forced Migration, Journal of Refugee Studies, 21 (4), pp. 432-453.

Bauman Zigmunt (2007) Consuming life, Cambridge; Malden, MA, Polity Press, $168 \mathrm{p}$.

Bauman Zigmunt (2000) Liquid modernity, London, Wiley-Blackwell, 240 p.

Bauman Zigmunt (1998) Globalization: The Human Consequences, New York, Columbia University Press, 160 p.

Becchi Egle e Julia Dominique (1996) Storia dell'infanzia: Dal Settecento a oggi, Bari-Roma, Laterza, 514 p.

Beneduce Roberto (2004) Frontiere dell'identità e della memoria: etnopsichiatria e migrazioni in un mondo creolo, Milano, FrancoAngeli, 320 p.

Beneduce Roberto, Frigessi Delia, Taliani Simona e Vacchiano Francesco (2000) Etnopsichiatria e migrazione: I'esperienza del Centro Frantz Fanon, in Pierluigi Morosini, Giovanni de Girolamo, Angelo Picaro e Gabriella Polidori Dir., La ricerca in salute mentale : risultati, implicazioni, ricadute, Roma, Laboratorio di Epidemiologia e Biostatistica, pp. 59-65.

Benslama Fethi (2004) La psychanalyse à l'épreuve de I'Islam, Paris, Flammarion, $334 \mathrm{p}$.

Biehl João, Good Byron and Kleinman Arthur (2007) Subjectivity: Ethnographic Investigations, Berkeley-Los Angeles-London, University of California Press, $477 \mathrm{p}$.

Carchedi Francesco (2009) II sistema di accoglienza. Modalità di intervento e criticità rilevate, in Giuliana Candia, Francesco Carchedi, Federica Giannotta e Giovanni Tarzia, Minori erranti. L'accoglienza e i percorsi di protezione, Roma, Ediesse, pp. 85-106.

Castles Stephen (2010) Understanding Global Migration: A SocialTransformation Perspective, Journal of Ethnic and Migration Studies, 36 (10), pp. 1565-1586.

Castles Stephen and Miller Marc J. (2009) The Age of Migration, Basingstoke, Palgrave Macmillan, $338 \mathrm{p}$.

Comaroff John and Comaroff Jean (2005) Reflections on Youth, from the Past to the Postcolony, in Filip De Boeck and Alcinda Honwana, Makers \& Breakers. Children \& Youth in Postcolonial Africa, Oxford-Trenton-Dakar, Currey-Africa World Press-Codesria, pp. 19-30. 
De Boeck Filip and Honwana Alcinda (2005) Children \& Youth in Africa. Agency, Identity \& Place, in Filip De Boeck and Alcinda Honwana, Makers \& Breakers. Children \& Youth in Postcolonial Africa, Oxford-Trenton-Dakar, Currey-Africa World Press-Codesria, pp. 1-18.

De Genova Nicholas P. (2002) Migrant Illegality and Deportability in Everyday Life, Annual Review of Anthropology, 31, pp. 419-447.

Derluyn Ilse and Broekaert Eric (2007) Different perspectives on emotional and behavioural problems in unaccompanied refugee children and adolescents, Ethnicity \& Health, 12 (2), pp. 141-162.

Dipartimento Giustizia Minorile (2008) Minori stranieri e giustizia minorile in Italia, Roma, Quaderni dell'Osservatorio sulla devianza minorile in Europa, $254 \mathrm{p}$.

Etiemble Angelina (2008) Parcours migratoires des mineurs isolés étrangers, catégorisation et traitement social de leur situation en France, e-migrinter, 2, pp. 180-185.

Fangen Katrine, Fossan Kirsten and Mohn Ferdinand A. (2010) Inclusion and Exclusion of Young Adult Migrants in Europe: Barriers and Bridges, London, Ashgate Publishing Ltd., 298 p.

Fass Paula S. (2005) Children in Global Migrations, Journal of Social History, $38(4)$, pp. 937-953.

Fass Paula S. (2003) Children and Globalization, Journal of Social History, 36 (4), pp. 963-977.

Fassin Didier (2011) Policing Borders, Producing Boundaries. The Governmentality of Immigration in DarkTimes, Annual Review of Anthropology, 40 (1), pp. 213-226.

Ferguson James (2006) Global Shadows: Africa in the Neoliberal World Order, Durham-London, Duke University Press, 272 p.

Ferguson James G. (2002) Of Mimicry and Membership: Africans and the "New World Society", Cultural Anthropology, 17 (4), pp. 551-569.

Fouquet Thomas (2007) Imaginaires migratoires et expériences multiples de I'altérité : une dialectique actuelle du proche et du lointain, Autrepart, 41 (1), pp. 83-98.

Frechaut Mónica (2007) A Situação dos Menores Desacompanhados em Portugal. Características e Recomendaçổes, Conselho Portugués para os Refugiados.

Furia Annalisa (2012) Victims or Criminals? The Vulnerability of Separated Children in the Context of Migration in the United Kingdom and Italy, Working papers of the Sussex Centre for Migration Research, 69, 30 p.

Gill Alan (1998) Orphans of the Empire: The Shocking Story of Child Migration to Australia, Sydney, Random House Australia, 701 p.

Giordano Carlo (2011) Le rôle de la famille d'origine dans le phénomène de la migration vers I'Italie de mineurs albanais non accompagnés par les parents ou les tuteurs, in Chantal Zaouche Gaudron Dir., Précarités et éducation familiale, Toulouse, Érès, pp. 327-331.

Giordano Carlo (2009) Les mineurs étrangers non accompagnés d'origine albanaise en Italie, Migrations société, 123, pp. 273-285. 
Giovannetti Monia (2009) Minori stranieri non accompagnati. Terzo rapporto $A N C l$, Roma, ANCl, 210 p.

Giovannetti Monia (2008) L'accoglienza incompiuta: le politiche dei comuni italiani verso un sistema di protezione nazionale per i minori stranieri non accompagnati, Bologna, II Mulino, 376 p.

Glick Schiller Nina and Salazar Noel B. (2013) Regimes of Mobility Across the Globe, Journal of Ethnic and Migration Studies, 39 (2), pp. 183-200.

Graw Knut and Schielke Samuli (2013) The Global Horizon: Migratory Expectations in Africa and Beyond, Leuven, Leuven University Press, 200 p.

Hannerz Ulf (1996) Transnational connections: culture, people, places, London-New York, Routledge, 216 p.

Heywood Colin (2001) A History of Childhood: Children and Childhood in the West from Medieval to Modern Times, Hoboken, Wiley Blackwell, 240 p.

Howes David (1996) Cross-cultural consumption: global markets, local realities, London, Routledge, $224 \mathrm{p}$.

Human Rights Watch (2013) Turned Away: Summary Returns of Unaccompanied Migrant Children and Adult Asylum Seekers from Italy to Greece, New York, Human Rights Watch, $45 \mathrm{p}$.

Human Rights Watch (2012) Boat Ride to Detention. Adult and Child Migrants in Malta, New York, Human Rights Watch, 50 p.

Human Rights Watch (2009) Perdus en zone d'attente. Protection insuffisante des mineurs étrangers isolés à l'aéroport de Roissy Charles de Gaulle, New York, Human Rights Watch, $67 \mathrm{p}$.

Human Rights Watch (2008) Left to Survive. Systematic Failure to Protect Unaccompanied Migrant Children in Greece, New York, Humans Rights Watch, $111 \mathrm{p}$.

Human Rights Watch (2002) Nowhere To Turn. State Abuses of Unaccompanied Migrant Children by Spain and Morocco, New York, Human Rights Watch, 62 p.

Human Rights Watch (1997) Slipping through the cracks: unaccompanied children detained by the U.S. Immigration and Naturalization Service, New York, Human Rights Watch, 139 p.

Jeffery Chris and Sherington Geoffrey (1998) Fairbridge: Empire and Child Migration, London-New York, Routledge, 304 p.

Laiz Moreira Sofia (2013) Itinerarios institucionalizados: Responsabilidades y dinámicas de exclusión en la protección de los menores migrantes no acompañados en Galicia, Revista Electrónica de Investigación y Docencia (REID), 0 (10), pp. 83-100.

Long Katy (2013) When refugees stopped being migrants: Movement, labour and humanitarian protection, Migration Studies, 1 (1), pp. 4-26.

Luhrmann Tanya Marie (2006) Subjectivity, Anthropological Theory, 6 (3), pp. 345-361.

Mbembe Achille (2000) À propos des écritures africaines de soi, Politique Africaine, 77, pp. 16-43. 
Mezzadra Sandro (2001) Diritto di fuga. Migrazioni, cittadinanza, globalizzazione, Verona, Ombre Corte, $133 \mathrm{p}$.

Miller Daniel (2012) Consumption and its consequences, Cambridge, Polity, 200 p.

Miller Daniel (2008) The comfort of things, Cambridge, Polity, 300 p.

Moore Henrietta L. (2007) The Subject of Anthropology: Gender, Symbolism and Psychoanalysis. Cambridge, Polity, 288 p.

Ortner Sherry B. (2005) Subjectivity and Cultural Critique, Anthropological Theory, 5 (1), pp. 31-52.

Palidda Salvatore (2008) Mobilità umane: introduzione alla sociologia delle migrazioni, Milano, Cortina, $211 \mathrm{p}$.

Pandolfo Stefania (2007) 'The burning'. Finitude and the Politico-Theological Imagination of Illegal Migration, Anthropological Theory, 7 (3), pp. 329-363.

Parousel Bernd (2011) Unaccompanied Minors in Europe: Between Immigration Control and the Need for Protection, in Gabriella Lazaridis, Security, Insecurity and Migration in Europe, London, Ashgate Publishing Ltd., pp. 139-160.

Quiroga Violeta, Ariadna Alonso y Montserrat Sòria (2010) Sueños de bolsillo. Menores Migrantes No Acompañados/as en España, Barcelona, Unicef-Banesto, $196 \mathrm{p}$.

Salazar Noel B. and Smart Alan (2011) Anthropological Takes on (Im)Mobility, Identities 18 (6), pp. i-ix.

Senovilla Hernández Daniel (2012) Analisi comparativa delle politiche comunitarie, dei contenuti della Convenzione sui Diritti del Fanciullo e delle prassi nazionali di accoglienza dei minori stranieri non accompagnati in Europa, in Luca Pacini e Paolo Testa, I minori stranieri non accompagnati in Italia. Quarto rapporto $\mathrm{ANCl}$, Roma, $\mathrm{ANCl}-\mathrm{Cittalia,} \mathrm{pp.} \mathrm{13-35.}$

Senovilla Hernández Daniel (2007) Situación y tratamiento de los menores extranjeros no acompañados en Europa, Bruxelles, Observatoire International de la Justice Juvénile, $62 \mathrm{p}$.

Sheller Mimi and Urry John (2006) The new mobilities paradigm, Environment and Planning $A, 38(2)$, pp. 207-226.

Sigona Nando (2011) Irregular Migrant Children and Public Policy, Oxford, COMPAS.

SOS Racismo y Colectivo Al Jaima (2005) Menores en las fronteras: de los retornos efectuados sin garantías a menores marroquíes y de los malos tratos subidos, Madrid-Tanger, Federación SOS Racismo.

Sourander Andre (1998) Behavior Problems and Traumatic Events of Unaccompanied Refugee Minors, Child Abuse \& Neglect, 22 (7), pp. 719-727.

Stearns Peter N. (2006) Consumerism in World History: The GlobalTransformation of Desire, London, Taylor \& Francis, 147 p.

Stearns Peter N. (2005) Change, Globalization and Childhood, Journal of Social History, 38 (4), pp. 1041-1046. 
Suárez Navaz Liliana (2006) Un nuevo actor migratorio: jóvenes, rutas y ritos juveniles transnacionales, in Francisco Checa, Angeles Arjona y Juan Carlos Checa Olmos, Menores tras la frontera. Otra migración que aguarda, Icaria, Antrazyt, pp. 17-50.

Suárez Navaz Liliana y Jiménez Alvarez Mercedes (2011) Menores en el campo migratorio transnacional. Los niños del centro (Drari d'sentro), Papers, Revista de Sociologia, 96 (1), pp. 11-33.

Taliani Simona e Vacchiano Francesco (2006) Altri corpi: antropologia ed etnopsicologia della migrazione, Milano, Unicopli, $336 \mathrm{p}$.

Unicef Maroc (2005) Nouveau visage de la migration. Les mineurs non accompagnés. Analyse transnationale du phénomène migratoire des mineurs marocains vers I'Espagne, Tanger, Litograph, $52 \mathrm{p}$.

Urry John (2007) Mobilities, Cambridge, Polity, 336 p.

Vacchiano Francesco (2012) Minori che migrano soli. Strategie di movimento e progetti di confinamento, in Stefano Volpicelli e Serena Saquella, Migrazione e Sviluppo: una nuova relazione?, Roma, Nuova Cultura, pp. 99-123.

Vacchiano Francesco (2010) Bash n'ataq I-walidin ("to save my parents"). Personal and social challenges of Moroccan unaccompanied children in Italy, in Joythi Kanics, Daniel Senovilla Hernández and Kristina Touzenis, Migrating Alone. Unaccompanied and Separated Children in Europe, Paris, Unesco Publishing, pp. 107-127.

Vacchiano Francesco (2008) "Bruciare di desiderio". Realtà sociale e soggettività dei giovani "harraga" marocchini, PhD Dissertation, Università degli Studi di Torino.

Vacchiano Francesco and Jiménez Mercedes (2012) Between agency and repression: Moroccan children on the edge, Children's Geographies, 10 (4), pp. 457-471.

Volpicelli Stefano (2012) L'attitude des jeunes au Maroc à l'égard de la migration : entre modernité et tradition. Réalisation d'un index de propension à la migration (IPM), Rabat, IOM-OIM, 80 p.

Werner Emmy E. (2009) Passages to America: Oral Histories of Child Immigrants from Ellis Island and Angel Island, Herndon, Potomac Books Inc., 208 p.

Whitehead Ann and Hashim Iman (2005) Children and Migration, Background Paper for DFID Migration Team, Sussex, University of Sussex, 57 p. 


\section{Francesco Vacchiano}

\section{À la recherche d'une citoyenneté globale. L'expérience des adolescents migrants en Europe}

La migration des " mineurs non accompagnés ", phénomène qui a pris de l'ampleur ces dernières années, met en évidence les transformations à la fois sociales et subjectives dans les contextes d'origine comme dans ceux de transit. À partir d'une recherche effectuée entre le Maghreb et l'Europe, I'auteur propose de traiter de l'expérience de la migration autonome d'enfants et d'adolescents comme d'une manifestation des formes hégémoniques d' être-au-monde " contemporain ; I'autonomie individuelle est liée au pouvoir de consommation, de communication et de mouvement. À travers leur désir d' "être modernes ", qui s'exprime par une demande de mobilité, les jeunes migrants "isolés " revendiquent une participation au monde d'aujourd'hui et une citoyenneté globale qui les projettent au-delà des frontières historiquement imposées.

\section{Searching for a Global Citizenship. Experience of Migrant Minors in Europe}

The recent increase of migratory flows that have children and adolescents as the main protagonists highlights the transformations, on a social and subjective level, in the contexts from where they are issued and which they cross. Basing the reflection on a research across the Maghreb and Europe, the author propose to understand the independent migration of children and adolescents as an expression of the contemporary, globally hegemonic forms of "being-in the-world". Such forms are moulded upon the achievement, by an autonomous subject, of the power of consumption, communication and movement. Through their desire of "being modern", specifically expressed through mobility, "separated" minors put across a request of participation to the contemporary world and a claim for global citizenship that project them beyond the historically imposed boundaries.

\section{En busca de una ciudadanía global. La experiencia de los menores migrantes en Europa}

La reciente intensificación de los flujos migratorios que implican a los menores como sujetos protagonistas nos lleva a interrogarnos sobre los cambios y las transformaciones sociales y subjetivas que conlleva esta migración en los contextos sociales de origen y de tránsito. A partir de mi investigación entre el Magreb y Europa, propongo une lectura de la migración independiente de niños y adolescentes como una manifestación de las formas hegemónicas contemporáneas de "estar en el mundo». Estas formas conjugan la autonomía individual con el poder de consumo, de comunicación y de movimiento. A través de su deseo de "ser modernos", los jóvenes migrantes "no acompañados» expresan, con la movilidad, una voluntad de participación en el mundo contemporáneo y una reivindicación de la ciudadanía global que los proyecta más allá de las fronteras históricamente impuestas. 